\title{
Drowsiness monitoring based on driver and driving data fusion
}

\author{
I. G. Daza, N. Hernandez, L. M. Bergasa, I. Parra, J. J. Yebes, M. Gavilan, \\ R. Quintero, D. F. Llorca, M. A. Sotelo \\ Departament of Electronics \\ University of Alcalá \\ Madrid, Spain \\ \{ivan.garcia,noelia.hernandez,bergasa \\ @ depeca.uah.es
}

\begin{abstract}
This paper presents a non-intrusive approach for monitoring driver drowsiness, based on driver and driving data fusion. The Percentage of Eye Closure (PERCLOS) is used to estimate the driver's state. The PERCLOS is computed on real time using a stereo vision-based system. The driving information used is the lateral position, the steering wheel angle and the heading error provided by the CAN bus. These three signals have been studied in the time and frequency domain. A multilayer perceptron neural network has been trained to fetch an optimal performance score. This system was installed in a naturalistic driving simulator. For evaluation purposes, several experiments were designed by psychologists and carried out with professional drivers. As ground truth, subjective experts' manual annotation of the driver video sequences and driving signals was used. A detection rate of $70 \%$ using individual indicators was raised up to $94 \%$ with the combination of indicators. An explanation about these results and some conclusion are presented.

Index Terms - Intelligent Transportation Systems, Driver Drowsiness, Confusion Matrix, PERcentage of eye CLOSure (PERCLOS), Visual Fatigue Behaviour, Artificial Neural Network, Spectral Density Power.
\end{abstract}

\section{INTRODUCTION}

Sleepiness during driving has been shown to result in a greatly increased risk of causing an accident. Specifically, Klauer et al. [1] have shown that to drive while sleepy increases the accident risk four to six times compared to alert driving. Furthermore, the risk of causing an accident is higher during night driving [2] or in situations with reduced prior sleep [3]. In fact, at least $15-20 \%$ of all vehicle accidents have been estimated to be sleepiness related [4]. Therefore, it is beneficial to develop a system to monitor the physical and mental state of the driver and give alerts at the critical moment when the driver is becoming fatigued, thereby preventing traffic accidents.

In the last decade, diverse techniques have been used to develop monitoring systems for a variety of purposes. Those techniques used to detect driver's sleepiness can be generally divided into three main categories [5]. The first category includes methods based on biomedical signals, like cerebral, muscular and cardiovascular activity [6], [7] and [8]. Usually, these methods require electrodes attached to the driver's body, which will often cause annoyance to the driver. Most of them are yet far from being effectively introduced in the market, according to recent reviews [9].
The second category includes methods based on driver performance, which evaluate variations in the lateral position of the vehicle, in the velocity, in the steering wheel angle and in other controller-area network (CAN) signals [10], [11] and [12]. The advantage of these approaches is that the signal is meaningful and the signal acquisition is quite easy. This is the reason why such systems have indeed entered the commercial market [13], [14] and [15] but, to the authors' knowledge, in the open literature there are very few details available regarding the mechanisms or parameters of these systems. On the other hand, these systems are subject to several limitations such as vehicle type, driver experience, geometric characteristics, condition of the road, etc. Then, these procedures require a considerable amount of time to analyse user behaviours and therefore, they do not work with the so called micro-sleeps-when a drowsy driver falls asleep for a few seconds on a very straight road section without changing the vehicle signals.

The third category includes methods based on driver visual analysis using image processing techniques. Computer vision can be a natural and non-intrusive technique for monitoring driver's sleepiness from the images taken by cameras placed in front of the user. These approaches are effective because the occurrence of sleepiness is reflected through the driver's face appearance and head/eyes activity. Different kinds of cameras and analysis algorithms have been reported in the literature for this approach: methods based on visible spectrum camera [16] and [17]; methods based on IR camera [5] and [18]; and methods based on stereo camera [19] and [20]. Some of them are commercial products as: Smart Eye [21], Seeing Machines DSS [22], Smart Eye Pro [19] and Seeing Machines Face API [20]. However, these commercial products are still limited to some well controlled environments and they require of hard calibration processes. Then, there is still a long way to go in order to obtain a robust commercial product in this category.

This paper present a non-intrusive approach for monitoring driver drowsiness, based on driver and driving data fusion. In Section II the simulator, the method used to study the fatigue and drowsiness in drivers and the generation of the ground truth signal are described. A general algorithm description, the techniques applied to obtain new indicators and the neural network designed is explained in Section III. After that, 
experimental results are shown in Section IV. Finally the conclusion and future work are presented in Section V.

\section{EXPERIMENTS AND DATA COLLECTION}

The purpose of this section is to show the characteristics of a naturalistic simulator and to explain the methodology carried out by psychologists in the analysis task to evaluate drowsiness in professional drivers.

\section{A. Realistic driving simulator}

Simulation methodologies applied to training and research in the field of road transport have been proven to be both cost-effective and efficient. Simulation aims to give the driver an opportunity to immerse himself in his habitual workplace. The study employed a naturalistic simulator placed at the CEIT [23], as we can see in Fig. 1.

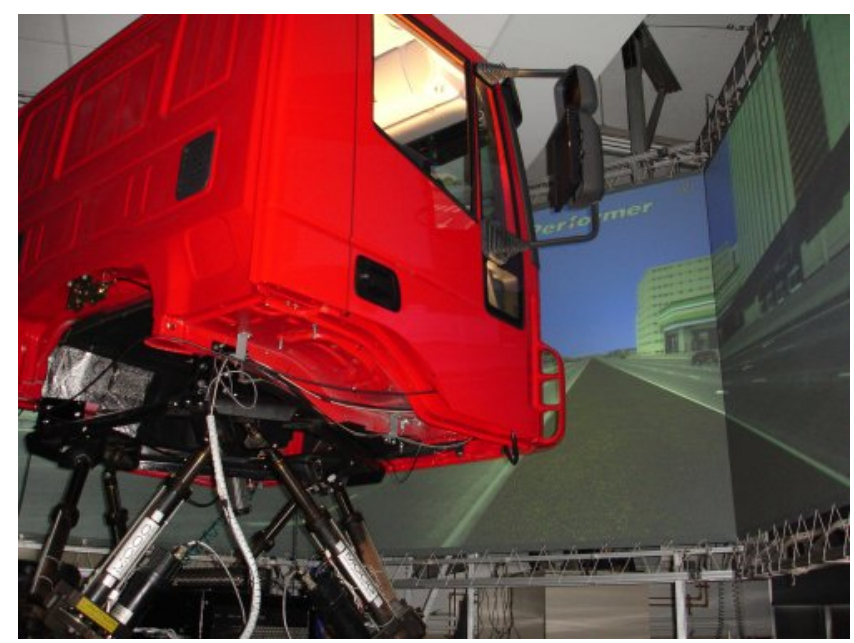

Fig. 1. Naturalistic simulator

The truck simulator consists of a real truck cab, an instructor station and a system of screens that cover 180 degrees of vision. A set of 3 screens with re-configurable positions cover a large area showing all angles of vision of drivers. The simulator has 3 scenarios (urban, interurban and mountain) with more than $250 \mathrm{~km}$ of roads, it allows driving in adverse conditions such as rain or snow, and at different times of day or night. The truck cab is an Iveco Stralis cab mounted on a Stewart platform 6GDL MOOG Company. The simulator records driving and driver variables. Concerning the driving variables the steering wheel angle, truck lateral position over the lane, heading error, speed, brake, acceleration and more variables are recorded to 120 samples per second. Within the driver variables the PERCLOS signal, blink frequency and others variables are recorded to 30 samples per second. Thus, this simulator gives the researcher great flexibility to implement recording systems specifically designed to test the reactions and behaviour of professional drivers while they are driving [24].

\section{B. Experimental protocol}

The main target of this experimental protocol is to recreate a suitable environment which would enable researchers to detect drowsiness during driving. A large amount of information about drowsiness clues have been gathered and analysed.

The data were collected for a total of 10 professional drivers. The protocol was designed such that each user would carry out driving sessions during two different conditions: Either after having slept on a regular schedule (from 23:00 to $07: 00$, with allowance for one hour deviations from the schedule) for two nights prior to the day of the experiment, or after having slept only four hours during the night preceding the experiment, thus being partially sleep deprived. Each user carried out driving sessions under each of the two conditions spread over a 24-hour period. Each driving session lasted 60 minutes. The tests subjects that participated in the study were recruited from the Spanish national register of professional drivers, by random selection (only private vehicle owners were included). They had to be frequent drivers, driving at least $5000 \mathrm{~km}$ a year, and not suffering from habitual sleep disturbances.

\section{Drowsiness ground truth}

The ground truth was labelled using the Karolinska Sleepiness Scale (KSS) and the video sequences. The test users were instructed to estimate their level of sleepiness according to the KSS, which has been proved to have a high correlation with a deterioration in driving performance which is closely related to the electroencephalography signal (EEG) [25]. The KSS level were subjectively generated by the driver every 5 minutes grouping the nine KSS levels into two: awake and drowsy. Finally this information was mixed with the three experts' offline analysis of the videos and the driving signals to generate the subjective ground truth signal in two levels: awake and drowsiness.

\section{DATA FUSION ALGORITHM TO DETECT DROWSINESS}

The general architecture of the implemented algorithm is shown in Fig. 2. The initial point is the collection of driver and driving related signals. The driver signal is the PERCLOS, that will be explained in Section III-A. The analysed driving signals are the lateral position, the steering wheel angle and the heading error. The driving signals are studied in the time III-A.1 and frequency III-A.2 domains to obtain new drowsiness indicators. In the time domain, the mean and standard deviation are used obtaining six indicators. In the frequency domain, the spectral density power is evaluated to find awake and drowsiness patterns. Finally, these indicators are combined in a multilayer perceptron neural network to estimate the drowsiness driver level.

\section{A. Input signals}

The driver signal employed is the Percentage of Eye Closure (PERCLOS), the most confident drowsiness indicator using computer vision algorithms [26] [5]. In order to obtain PERCLOS a NIR stereo rig is placed in front of the driver. The system works in real-time and does not need a 


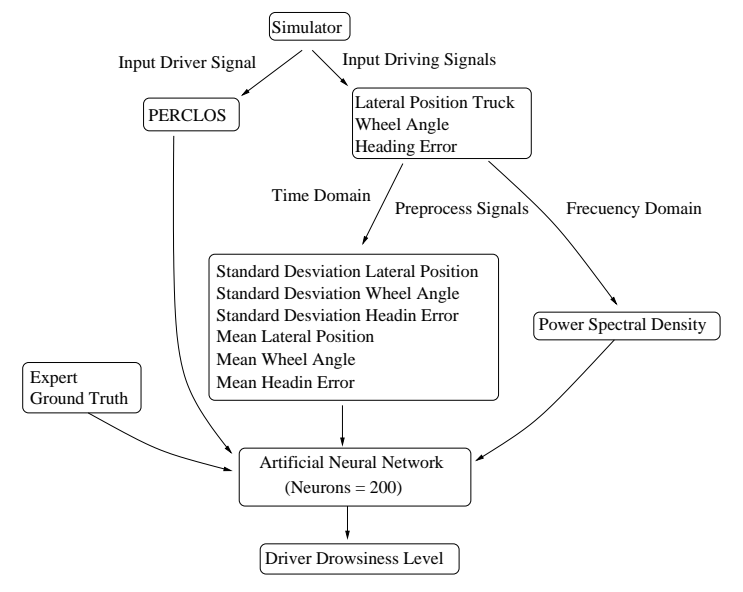

Fig. 2. General architecture

calibration process. The system consists in three main stages. The first one is the pre-processing stage, which includes face and eye detection based on appearance strategy using the Viola and Jones algorithm [27], and the equalization of the eyes using a Hat transformation. An eye tracking strategy in a sequence of frames is then carried out. The second one executes the pupil position extraction and its characterization using integral projection techniques and a Gaussian model. The final stage executes the PERCLOS estimation [26]. The PERCLOS is defined as the percentage for a given period of time (here set to 20 seconds) for which eyes are at least $80 \%$ covered by eyelids. This measure has been found to correlate well with reduced psycho-motor vigilance task and deteriorating driving performances due to sleepiness.

The driving signals are the lateral position, the steering wheel and the heading error angle of the truck simulator. These were obtained from the CAN bus. The lateral position represents the distance from the centre of the vehicle to the driving right lane boundary. The steering wheel angle represents the wheel movements and the heading angle is the angle between the direction of the vehicle with the tangent to the path.

The PERCLOS and lateral position parameters without preprocessing are represented as a function of distance in Fig. 3 for two different experiments: one in which the driver was awake and one in which showed drowsiness. A time representation is the regular form to show the results in this studies. However, in this paper we choose as a common line reference for these two variables the distance in $\mathrm{km}$, which means to take the same stretch trajectory for the same driver and under different drowsiness condition. On top plot in Fig. 3, the PERCLOS signal clearly shows higher values for the drowsiness experiment. Besides, the lower plot represents the lateral position signal, where it can be observed that the amplitude and dispersion of its values are greater for the drowsiness case. Hence, it is very convenient to analyze these measurements to detect driver sleepiness.

1) Time domain pre-process: Many indicators of driver sleepiness have been proposed in the literature [28]; regres-
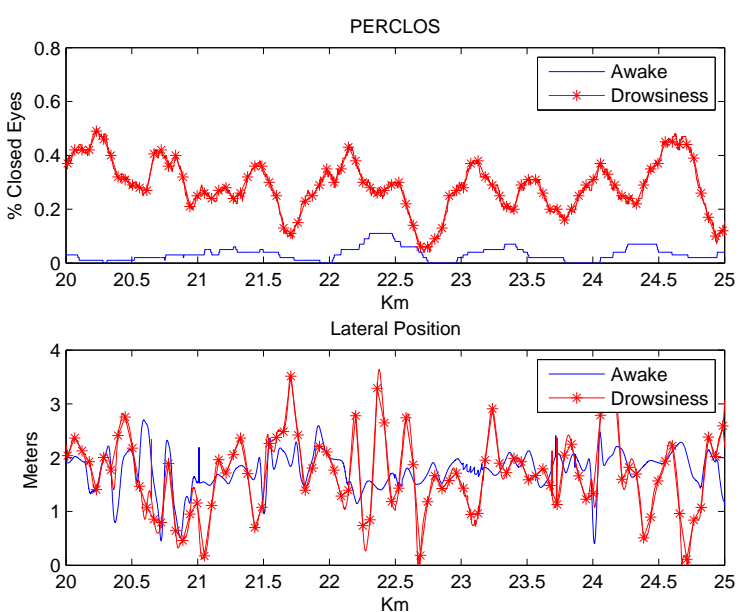

Fig. 3. Input variables

sion descriptors, class distribution measures, peak amplitudes and distances, but perhaps the most common indicator is the standard deviation of the lateral position which simply measures the average lateral deviation.

In this paper, we compute the standard deviation and mean over the values of the following signals: lateral position, steering wheel angle and heading error. These new statistical indicators have been evaluated with different temporal windowing sizes in order to obtain the best value for this application.

The mean indicators do not show evidence about drowsiness with different windowing size, but it will be used to evaluate the neural network performance. However the standard deviation applied to the lateral position and steering wheel angle show differences between awake and drowsiness when the windowing size is 1000 meters, as we depict in Fig. 4. On top, the standard deviation of lateral position shows that its values in the drowsiness exercise are higher than in the awake one. However on the bottom subfigure the standard deviation of steering wheel angle does not show large differences between the drowsiness and the awake exercise. In the drowsiness exercise the values of standard deviation are a bit larger than in the awake one. These differences are explained because the driving signals have more amplitude and the car corrections are less accurate when the driver is drowsy.

2) Frequency domain pre-process: This study is performed to find more indicators to determine if the driver is in an awake or drowsiness state because in the time domain the mean and standard deviation indicators of steering wheel angle and heading error do not show evidence of drowsiness, as explained before.

The idea is that an awake driver performs continuous corrections on the steering wheel when the vehicle is under control. On the contrary, a drowsy driver does not perform continuous corrections, and chaotic movements are detected on the steering wheel movements. The expected fatigue 

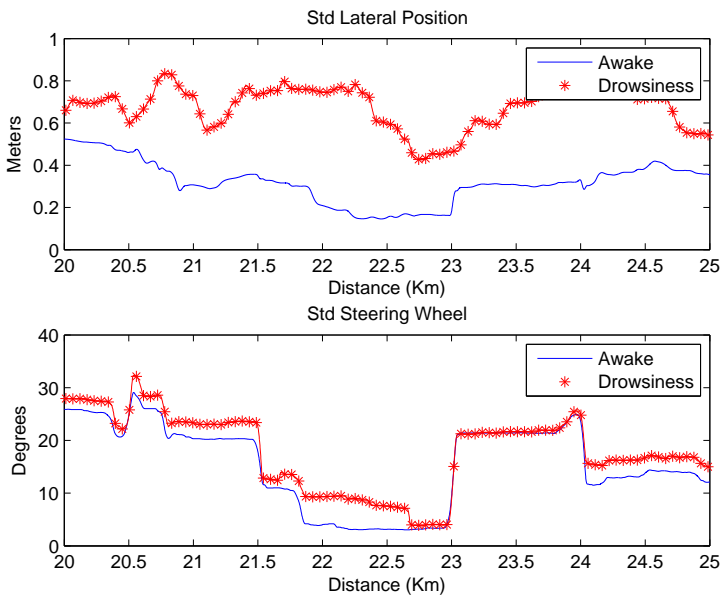

Fig. 4. Indicators obtained with the standard deviation of the lateral position and steering wheel angle

induced changes in steering wheel behaviour are a pattern of slow drifting and fast corrective counter steering, too.

The spectral energy distribution has been evaluated with a Fast Fourier Transform (FFT) and a window size of 500 meters to evaluate the energy from $0.01 \mathrm{~Hz}$ to $0.03 \mathrm{~Hz}$. Therefore, a concentrated spectral energy is found in the awake state, while the drowsiness state is characterized by an extended spectral energy distribution. This is explained by the chaotic movements and the different frequencies in the steering wheel movements as shown in Fig. 5.
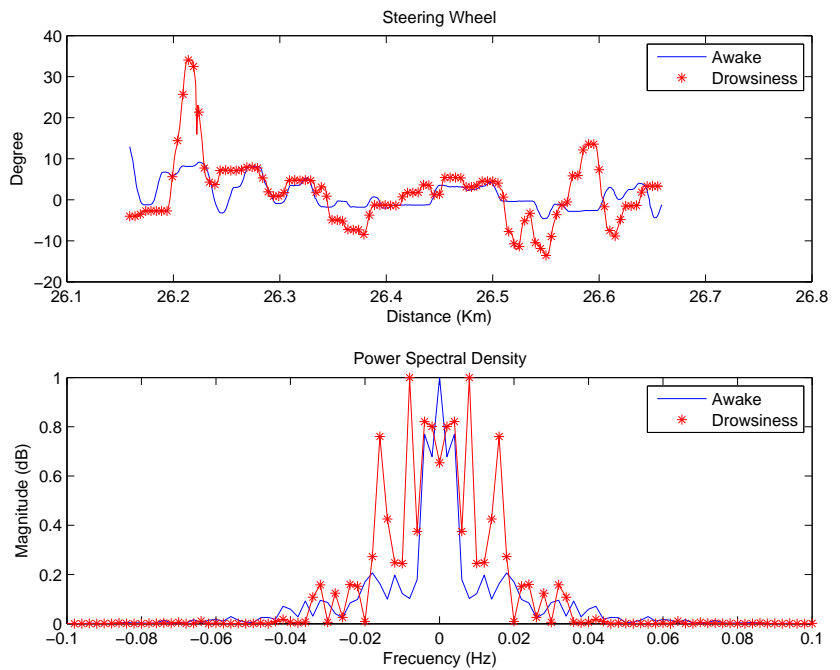

Fig. 5. Steering wheel power spectral density

To make the path of the road independent with steering movements of the route due to curves, the heading error has been introduced. This variable shows the same effects than the steering wheel movements: oscillations occur when the vehicle is being controlled, while oscillations disappear or are chaotic when the driver loses the control.

\section{B. Neural Network Designed}

A combination of several indicators may provide a better estimate of sleepiness than any individual indicator alone. The indicators can be combined in several ways, ranging from simple linear combination of indicator values to much more complex functional forms. In order to capture as many functional forms as possible, artificial neural networks (ANN) have been used here. We have used a two layered feed-forward ANN as depicted in Fig. 6.

The strategy for training has been to divide the set of inputs into three separate data sets: training set (used to guide the parameter search carried out by the optimization algorithm), validation set (used for determining when to terminate the optimization) and test set (used to obtain the performance on previously unseen data). The total number of samples per user are 30000 , equivalent to the total number of exercise meters. The training, validation and test samples are grouped in windows of size 100 . Hence, considering all the users there are 3000 total input samples to adjust the weights in the network. Therefore, the number of inputs to the neural network is 100 when used only one indicator and 200 inputs for two indicators. Thus, the system will alert the driver every 100 meters.

The network configuration is based on Tan-Sigmod Transfer Function for hidden neuron layer and a Linear Transfer Function for output layer neurons. The number of neurons in the input layer is a function of the input variables used. On the other hand, the number of neurons in the hidden layer is set to 20 due to it is recommended for no more than $10 \%$ of the inputs. The target training has been set that the mean square error will be below of 0.01 or the number of epochs will be less than 200 . The backpropagation algorithm is used to train the network and the gradient descent is applied to adjust the weights in the network.

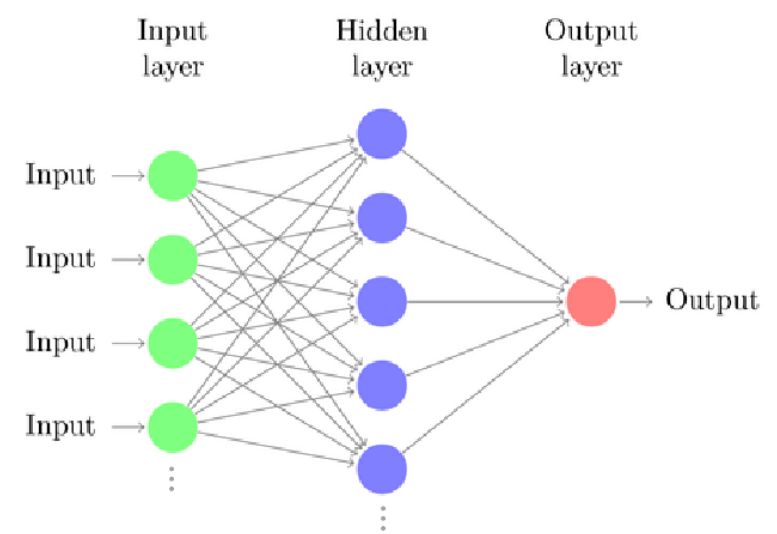

Fig. 6. Neural network designed

\section{EXPERIMENTAL RESULTS}

The designed network was discussed in Section III-B and the inputs and outputs signals used to train the artificial neural network were specified in Section III-A. The 
detection rate has been evaluated with 12 different input indicators. The detector rate is evaluated with the expression 1 -mean square error (mse). These 12 indicators are used alone and in combinations of them. The alone indicator study is done with PERCLOS, lateral position (Lp), mean lateral position (Avg Lp), standard deviation lateral position (Std Lp), steering wheel (Wheel), mean steering wheel (Avg Wheel), standard deviation steering wheel (Std Wheel), heading error (He), mean heading error (Avg He), standard deviation heading error (Std He), wheel energy (Wheel Pow) and heading error energy (He Pow) indicators. The alone indicators detection rate is shown in Table I.

TABLE I

Single DETECTION RATE

\begin{tabular}{c|c|c|c}
\hline & Single & Mean & Std \\
\hline \hline Lateral Position & 66.71 & 64.10 & 84.10 \\
\hline \hline Steering Wheel & 40.57 & 58.21 & 64.10 \\
\hline \hline Heading Error & 60.04 & 61.51 & 62.92 \\
\hline \hline Energy Wheel & 33.51 & No data & No data \\
\hline \hline Energy He & 62.14 & No data & No data \\
\hline \hline PERCLOS & 97.61 & No data & No data \\
\hline
\end{tabular}

Among the driving signals, in the time domain, the lateral position indicator provides the higher detection rate values while the steering wheel angle and heading error provide similar results. The standard deviation of all these indicators provide better results than the others and a $84.10 \%$ is the best detection rate obtained with the standard deviation of the lateral position. On the other hand, in the frequency domain, the wheel and the energy do not yield high detection rates, with a $33.52 \%$ and $62.14 \%$ respectively. The results obtained are not better than in the time domain. Looking at the driver signal, the PERCLOS is the best with a $97.61 \%$ of detection rate. The improvement is mainly due to independence of the PERCLOS signal with the trajectory. These rate results could be higher with a combination of indicators. The indicators with a detection rate higher than $62 \%$ in Table I are selected to be combined two by two. The new detection rate obtained is represented on table II. The table represents the combination between the rows and the columns indicators. More than one input produce generally higher hit rates than those that use only single inputs. In our case the combination of energy indicators with other driving or driver signal produced a decrease of the hit rate due to the continuous changes in energy distribution frequency during the exercises. The combination of the PERCLOS with other indicators obtained the best results and improved its single detection rate. The combination between the PERCLOS and standard deviation of the lateral position produced the best results with a $98.65 \%$ of detection rate, that is above the results obtained in [29] where data collection is similar to that used in this paper, a total of 14 drivers is used and they were given the task to drive under two different conditions: sleep-deprived and after normal sleep. So these two variables will be very important to design a driver drowsiness detection system with neural networks or learning algorithms.
TABLE II

COMBINATION DETECTION RATE

\begin{tabular}{c|c|c|c|c|c|c}
\hline & Lp & Avg Lp & Std Lp & Std Wheel & Std He & Ener. He \\
\hline \hline PERCLOS & 98.60 & 97.34 & 98.65 & 97.95 & 98.60 & 64.09 \\
\hline \hline Lp & & 82.91 & 67.62 & 59.39 & 74.68 & 64.74 \\
\hline \hline Avg Lp & & & 93.51 & 64.10 & 74.68 & 39.39 \\
\hline \hline Std Lp & & & & 87.63 & 75.86 & 31.15 \\
\hline \hline Std Wheel & & & & & 67.63 & 38.21 \\
\hline \hline Std He & & & & & & 46.18 \\
\hline
\end{tabular}

The detailed confusion matrix of the neural networks classification results for the PERCLOS-standard deviation lateral position and PERCLOS-lateral position combination are given in Table III and IV.

TABLE III

CONFUSION MATRIX PERCLOS-STANDARD DEVIATION LATERAL POSITION

\begin{tabular}{cc|cc}
\hline & \multicolumn{2}{|c}{ Correct } \\
& & Awake & Drowsiness \\
\hline \hline \multirow{2}{*}{ Estimated } & Awake & $100 \%$ & $6.25 \%$ \\
& Drowsiness & $0 \%$ & $93.75 \%$ \\
\hline
\end{tabular}

TABLE IV

CONFUSION MATRIX PERCLOS-LATERAL POSITION

\begin{tabular}{lc|cc}
\hline & \multicolumn{2}{|c}{ Correct } \\
& & Awake & Drowsiness \\
\hline \multirow{2}{*}{ Estimated } & Awake & $100 \%$ & $9.37 \%$ \\
& Drowsiness & $0 \%$ & $90.62 \%$ \\
\hline
\end{tabular}

\section{CONCLUSIONS AND FUTURE WORKS}

This paper presents a non intrusive approach for monitoring driver drowsiness, based on driver and driving information and data fusion, and has been tested in a naturalistic driving simulator. The proposed drowsiness detection method has shown high accuracy, obtaining $98.65 \%$ of drowsiness detection rate.

In general, PERCLOS can be used in combination with lateral position and steering wheel angle, as they provide complementary information, to improve the drowsiness monitoring in drivers. The standard deviation of the lateral position or steering wheel angle have been proved to be the best signals to fuse with the PERCLOS obtaining the highest detection results. The heading error and the energy signals do not improve the hit rate because they do not present correlation with the drowsiness pattern.

The results shown in this paper are influenced by the simulator used. A good way to measure how robust is the algorithm presented is to apply it under real world conditions. In addition, generating a ground truth drowsiness signal by expert psychologists is important to evaluate the hit rate of any method related with drowsiness detection.

As future work we envision the use of Hidden Markovmodel and Bayesian networks to model temporal aspects and experts knowledge. Also a multi-level classification to adapt 
varying driving styles and road conditions could improve the current detection rates. Finally, exhaustive tests in real conditions with more drivers should be performed to evaluate the effect of the driving styles and the driving conditions in the detector performance.

\section{ACKNOWLEDGEMENTS}

This work has been financed with funds from the Ministerio de Ciencia e Innovación through the project DRIVERALERT (TRA2008 - 03600), as well as from the project CABINTEC (PSE-370000-2009-12).

\section{REFERENCES}

[1] S. G. Klauer, T. A. Dingus, V. L. Neale, , and J. D. Sudweeks, "The impact of driver inattention on near-crash/crash risk: An analysis using the 100-car naturalistic driving study data," National Highway Traffic Safety Administration, DC, DOT HS, vol. 810, 2006.

[2] T. Akerstedt, G. Kecklund, and L. Hörte, "Night driving, season, and the risk of highway accidents." Sleep, vol. 24, pp. 401-406, 2001.

[3] J. Connor, R. Norton, S. Ameratunga, E. Robinson, I. Civil, R. Dunn, J. Bailey, and R. Jackson, "Driver sleepiness and risk of serious injury to car occupants: Population based control study." British Medical Journal, vol. 324, pp. 1125-1129, 2002.

[4] J. Horne and L. Reyner, "Vehicle accidents related to sleep: A review." Occupational and Environmental Medicine, vol. 56, pp. 189-294, 1999.

[5] L. M. Bergasa, J. Nuevo, M. Á. Sotelo, R. Barea, and M. E. L. Guillén, "Real-time system for monitoring driver vigilance," IEEE Transactions on Intelligent Transportation Systems, vol. 7, no. 1, pp. 63-77, 2006.

[6] T. Oron-Gilad, A. Ronen, and D. Shinar, "Alertness maintaining tasks (amts) while driving," Accident Analysis \& Prevention, vol. 40, no. 3, pp. 851-860, May 2008.

[7] C. Papadelis, Z. Chen, C. Kourtidou-Papadeli, P. Bamidis, I. Chouvarda, E. Bekiaris, and N. Maglaveras, "Monitoring sleepiness with on-board electrophysiological recordings for preventing sleep-deprived traffic accidents." Clinical Neurophysiology, vol. 118, no. 9, pp. 19061922, September 2007.

[8] J. Faber, "Detection of different levels of vigilance by eeg pseudo spectra." Neural Network World, vol. 14, no. 3-4, pp. 285-290, 2004.

[9] N. Wright, B. Stone, T. Horberry, and N. Reed, "A review of in-vehicle sleepiness detection devices," TRL Limited, Published Project Report PPR157, 2007.

[10] T. Wakita, K. Ozawa, C. Miyajima, K. Igarashi, K. Itou, K. Takeda, and F. Itakura, "Driver identification using driving behavior signals," IEICE - Trans. Inf. Syst., vol. E89-D, no. 3, pp. 1188-1194, 2006.

[11] Y. Takei and Y. Furukawa, "Estimate of driver's fatigue through steering motion." IEEE International Conference on Systems, Man and Cybernetics., vol. 2, pp. 1765-1770, 2005.

[12] J. C. McCall, M. M. Trivedi, D. Wipf, and B. Rao, "Lane change intent analysis using robust operators and sparse bayesian learning," in CVPR '05: Proceedings of the 2005 IEEE Computer Society Conference on Computer Vision and Pattern Recognition (CVPR'05) - Workshops. Washington, DC, USA: IEEE Computer Society, 2005, p. 59.

[13] "Volvo car corporation," http://www.media.volvocars.com.

[14] "Mercedes-benz," http://www.emercedesbenz.com/Aug08.

[15] "Lexus," http://www.testdriven.co.uk/lexus-1s-600h.

[16] T. D'Orazio, M. Leo, C. Guaragnella, and A. Distante, "A visual approach for driver inattention detection," Pattern Recogn., vol. 40, no. 8, pp. 2341-2355, 2007.

[17] M. Suzuki, N. Yamamoto, O. Yamamoto, T. Nakano, and S. Yamamoto, "Measurement of driver's consciousness by image processing -a method for presuming driver's drowsiness by eye-blinks coping with individual differences." Systems, Man and Cybernetics, vol. 4, pp. 2891-2896, 2006.

[18] Q. Ji and X. J. Yang, "Real-time eye, gaze, and face pose tracking for monitoring driver vigilance," Real-Time Imaging, vol. 8, no. 5, pp. 357-377, 2002.

[19] "Smart eye,"

http://www.smarteye.se.
[20] "Seeingmachines," http://www.seeingmachines.com.

[21] L. Bretzner and M. Krantz, "Towards low-cost systems for measuring visual cues of driver fatigue and inattention in automotive applications," Vehicular Electronics and Safety. IEEE International Conference, pp. 161-164, 2005.

[22] J. Heinzmann, D. Tate, and R. Scott, "Using technology to eliminate drowsy driving," SPE International Conference on Health, Safety, and Environment in Oil and Gas Exploration and Production, April 2008.

[23] "Tutor [online]," http://www.landersimulation.com/index.php.

[24] B. Lang, A. Parkes, S. Cotter, R. Robbins, C. Diels, P. Vanhulle, G. Turi, E. Bekiaris, M. Panou, and J. K. . S. Poschadel, "Train-all integrated system for driver training and assessment using interactive education tools and new training curricula for all modes of road transport," 2007.

[25] K. Kaida, M. Takahashi, T. Akerstedt, A. Nakata, Y. Otsuka, T. Haratani, and K. Fukasawa, "Validation of the karolinska sleepiness scale against performance and eeg variables."

[26] I. Garcia, S. Bronte, L. Bergasa, N. Hernandez, B. Delgado, and M. Sevillano, "Vision-based drowsiness detector for a realistic driving simulator," in Intelligent Transportation Systems (ITSC), 2010 13th International IEEE Conference on Intelligent Transportation Systems, 2010, pp. 887-894.

[27] P. Viola and M. J. Jones, "Robust real-time face detection," Int. J. Comput. Vision, vol. 57, no. 2, pp. 137-154, 2004.

[28] D. Sandberg, T. Akerstedt, A. Anund, G. Kecklund, and M. Wahde, "Detecting driver sleepiness using optimized non-linear combinatioins of sleepiness indicators," in IEEE Transactions on Intelligent Transportation Systems, vol. 12, no. 1, 2011, pp. 97-108.

[29] D. Sandberg and M. Wahde, "Particle swarm optimization of feedforward neural networks for the detection of drowsy driving," in Neural Networks, 2008. IJCNN 2008. (IEEE World Congress on Computational Intelligence). IEEE International Joint Conference on Neural Networks., 2008, pp. 788-793. 